Research Article

\title{
Observation of Dissipative Bright Soliton and Dark Soliton in an All-Normal Dispersion Fiber Laser
}

\author{
Chunyang Ma, ${ }^{1}$ Bo Gao, ${ }^{2}$ Ge Wu, ${ }^{1}$ Tian Zhang, ${ }^{1}$ and Xiaojian Tian' \\ ${ }^{1}$ College of Electronic Science and Engineering, Jilin University, Changchun 130012, China \\ ${ }^{2}$ College of Communication Engineering, Jilin University, Changchun 130012, China \\ Correspondence should be addressed to Xiaojian Tian; tianxj@jlu.edu.cn
}

Received 22 June 2016; Revised 19 August 2016; Accepted 22 August 2016

Academic Editor: Xiaohui Li

Copyright (c) 2016 Chunyang Ma et al. This is an open access article distributed under the Creative Commons Attribution License, which permits unrestricted use, distribution, and reproduction in any medium, provided the original work is properly cited.

\begin{abstract}
This paper proposes a novel way for controlling the generation of the dissipative bright soliton and dark soliton operation of lasers. We observe the generation of dissipative bright and dark soliton in an all-normal dispersion fiber laser by employing the nonlinear polarization rotation (NPR) technique. Through adjusting the angle of the polarizer and analyzer, the mode-locked and non-modelocked regions can be obtained in different polarization directions. Numerical simulation shows that, in an appropriate pump power range, the dissipative bright soliton and dark soliton can be generated simultaneously in the mode-locked and non-mode-locked regions, respectively. If the pump power exceeds the top limit of this range, only dissipative soliton will exist, whereas if it is below the lower bound of this range, only dark soliton will exist.
\end{abstract}

\section{Introduction}

With the rapid development of ultrafast optical field, a great many researchers have been focusing on mode-locked fiber laser recently [1-7]. Passive mode-locked fiber lasers have shown many advantages over solid-state systems such as the compact design, low-cost, and stability [8-11]. It has also some potential applications, like laser processing, optical communications, medical equipment, military, and so on [12-14]. Ultrashort pulses are stabilized in an oscillator when the effects of optical nonlinearity are exactly balanced by other processes after one cycle around the cavity. The most widely used method to compensate nonlinearity is group velocity dispersion (GVD). The traditional soliton is formed by the balance between nonlinear and negative dispersion phase changes. The resulting soliton propagates indefinitely without change. However, the traditional soliton is limited to $0.1 \mathrm{~nJ}$ of the energy in standard fibers [15]. When the GVD reaches zero, the dispersion managed soliton has been formed, which enables emitting the pulses with the pulse energy reaching the $1 \mathrm{~nJ}$ level [16]. Instead of conventional soliton and dispersion managed soliton, self-similar soliton can tolerate strong nonlinearity without wave breaking. But due to the restricted gain bandwidth, self-similar soliton allows the energy to reach the $10 \mathrm{~nJ}$ level [17]. Dissipative soliton (DS) has attracted great interest in the development of fiber lasers because it can significantly improve the deliverable energy of pulse, approaching or even exceeding $100 \mathrm{~nJ}$ $[18,19]$, and the DS exists in nonconservative systems whose dynamics are extremely different from those of conventional soliton [20-22]. A fiber laser with pure normal GVD (or large normal GVD together with small anomalous GVD) would presumably have to exploit dissipative process in the modelocked pulse shaping [23-25].

The research of dark soliton lags behind that of bright soliton due to the difficulty of dark soliton generation. However, dark soliton is more suitable than bright soliton when used in optical communications [26]. Dark soliton is broadened during propagation at nearly half the rate of bright soliton, and dark soliton shows more resistance than bright soliton to perturbation during propagation. Furthermore, as the background noise mainly affects the background of the dark soliton, it is less sensitive to background noise [2729]. Recently, the dark soliton formation in fiber lasers has been reported in literature [30-33]. Zhang et al. have experimentally observed dark soliton in a fiber ring laser with all-anomalous dispersion fibers [30] and with all-normal dispersion fibers [31]. Tang et al. demonstrated the dark 
soliton formation in an all-normal dispersion cavity fiber laser without an antisaturable absorber in cavity [33].

In this paper, we propose a novel way for controlling the generation of the dissipative bright soliton and dark soliton operation of lasers. The nonlinear polarization rotation technique is implemented for generating mode-locked and nonmode-locked region. The dissipative soliton and dark soliton are obtained in mode-locked and non-mode-locked regions, respectively.

\section{Modeling}

To study the feature and dynamic evolution of dissipative and dark soliton in an all-normal dispersion fiber laser, we implement a numerical model that incorporates the most important physical effects like the nonlinear polarization rotation (NPR), spectral filtering (SF), and so forth. The propagation model is shown schematically in Figure 1. It consists of a $4 \mathrm{~m}$ erbium-doped fiber (EDF) and $1.8 \mathrm{~m}$ dispersion compensating fiber (DCF), and the polarization additive-pulse modelocking (PAPM) system is made of a polarization-sensitive isolator and two sets of polarization controllers, two quarter waveplates (QWP), and a half waveplate (HWP) made of the polarizer; one QWP and a HWP constitute the analyzer. The PAPM system is used to produce the NPR effect, which relies on the intensity dependent rotation of an elliptical polarization state in a length of optical fiber. By setting the angle of the polarizer and the linear cavity phase delay of the cavity appropriately, we can obtain the mode-locked and the non-mode-locked area in PAPM where mode-locked area means that the light intensity is inversely proportional to the loss and the opposite non-mode-locked area. When the soliton propagates through the PAPM element and the intensity transmission, $T$ is expressed as [11]

$$
\begin{aligned}
T= & \sin ^{2}(\theta) \sin ^{2}(\varphi)+\cos ^{2}(\theta) \cos ^{2}(\varphi) \\
& +0.5 \sin (2 \theta) \sin (2 \varphi) \cos \left(\Delta \phi_{L}+\Delta \phi_{N L}\right),
\end{aligned}
$$

where $\theta$ and $\varphi$ represent the angle between the fast axis of the birefringent fiber and polarizer (analyzer), $\Delta \phi_{L}$ is the linear cavity phase delay, $\Delta \phi_{L}=(2 \pi L / \lambda)\left(n_{x}-n_{y}\right)+\phi_{P C}, \phi_{P C}$ is the phase difference between two polarization directions, and $\lambda$ and $L$ represent the cavity length of the fiber and the wavelength of light, respectively. $\Delta \phi_{N L}$ is the nonlinear phase delay caused by self-phase modulation (SPM) and cross-phase modulation (XPM). $\Delta \phi_{N L}=-\left(2 \pi L n_{2} P / 3 \lambda A_{\text {eff }}\right) \cos 2 \alpha, n_{2}$ is the nonlinear coefficient, $A_{\text {eff }}$ is the effective mode area, and $P$ is optical power.

The pulse propagation in the weak birefringent fiber can be modeled well by two coupled nonlinear Schrodinger equations (NLSE). However, the fiber lasers have components which cannot be modeled by several phase modulation terms in NLSEs. For example, a fiber laser has a gain with a limited gain bandwidth (BW) and a saturable absorber (SA). They do not induce the phase modulation, but they definitely cause the amplitude modulation in the frequency and time domain. To count the amplitude modulation effects in a laser cavity properly, more terms needed to be added to
NLSEs. The resulting differential equations are called coupled cubic Ginzburg-Landau equations (CGLE) [34], shown as

$$
\begin{aligned}
& \frac{\partial u_{x}}{\partial z}+\frac{\alpha}{2} u_{x}+\delta \frac{\partial u_{x}}{\partial T}+i \frac{\beta_{2}}{2} \frac{\partial^{2} u_{x}}{\partial T^{2}}-\frac{g}{2} u_{x}-\frac{g}{2 \Omega_{g}^{2}} \frac{\partial^{2} u_{x}}{\partial T^{2}} \\
& =i \gamma\left(\left|u_{x}\right|^{2}+\frac{2}{3}\left|u_{y}\right|^{2}\right) u_{x}, \\
& \frac{\partial u_{y}}{\partial z}+\frac{\alpha}{2} u_{y}-\delta \frac{\partial u_{y}}{\partial T}+i \frac{\beta_{2}}{2} \frac{\partial^{2} u_{y}}{\partial T^{2}}-\frac{g}{2} u_{y}-\frac{g}{2 \Omega_{g}^{2}} \frac{\partial^{2} u_{y}}{\partial T^{2}} \\
& =i \gamma\left(\left|u_{y}\right|^{2}+\frac{2}{3}\left|u_{x}\right|^{2}\right) u_{y},
\end{aligned}
$$

where $u_{x}$ and $u_{y}$ denote the envelopes of the optical pulses along the two orthogonal polarization axes of the fiber, and $\alpha$ is the loss coefficient of the fiber. $\delta=\left(\beta_{1 x}-\beta_{1 y}\right) / 2$ is the group velocity difference between the two polarization modes, $\beta_{2}$ represents the fiber dispersion, $\Omega_{g}$ is the bandwidth of the laser gain, $T=t-\left(\beta_{1 x}+\beta_{1 y}\right) z / 2$ and $z$ indicate the pulse local time and the propagation distance, respectively, and $g$ describes the gain function of EDF which is expressed by $g=g_{0} \exp \left(-E_{\text {pulse }} / E_{\text {sat }}\right)[2]$, where $g_{0}$ is the small signal gain coefficient, related to the doping concentration, and $E_{\text {sat }}$ is the gain saturation energy, which corresponds to the pumping strength $[2,34]$. The pulse energy $E_{\text {pulse }}$ is given by $E_{\text {pulse }}=$ $\int_{-T_{R} / 2}^{T_{R} / 2}\left(|u|^{2}+|v|^{2}\right) d \zeta$, where $T_{R}$ is the cavity round-trip time.

\section{Simulation Results and Discussion}

The model is solved with a standard symmetric split-step Fourier algorithm. The initial $u_{x}$ denotes an arbitrary signal, while the initial $u_{y}$ denotes a weak continuous wave (CW) light which contains a small segment dip on the top of it; by appropriately adjusting the angle of the polarizer (analyzer) and the linear cavity phase delay of the cavity, both dissipative and dark solitons can be achieved in different directions of the polarization. Since the saturation energy $E_{\text {sat }}$ is proportional to the pumping strength [35], this means increasing $E_{\text {sat }}$ corresponds to increasing the pump power in the practical system. The following parameters are applied for the simulations possibly matching the experimental conditions: $\alpha=$ $0.2 \mathrm{~dB} / \mathrm{km}, g_{0}=2 \mathrm{~m}^{-1}, \gamma=4.2 \mathrm{~W}^{-1} \mathrm{~km}^{-1}$ for EDF and $\gamma=1.3 \mathrm{~W}^{-1} \mathrm{~km}^{-1}$ for DCF, $\Omega_{g}=30 \mathrm{~nm}, \mathrm{EDF}=4.0 \mathrm{~m}$, $\mathrm{DCF}=1.8 \mathrm{~m}, \beta_{2}=+31 \times 10^{-3} \mathrm{ps}^{2} / \mathrm{m}$ for EDF and $\beta_{2}=+20 \times$ $10^{-3} \mathrm{ps}^{2} / \mathrm{m}$ for DCF, and net cavity GVD $\beta_{\text {net }} \approx 0.16 \mathrm{ps}^{2}$, $\theta=\pi / 8, \varphi=5 \pi / 8, \phi_{P C}=0.96 \pi$, and when $E_{\text {sat }}=460 \mathrm{pJ}$, it is capable of achieving both dissipative and dark solitons in fiber laser.

The formation and evolution of dissipative and dark solitons at $E_{\text {sat }}=460 \mathrm{pJ}$ are illustrated in Figure 2. We can find that the dissipative soliton pulse duration, pulse energy, and peak power are about $2.96 \mathrm{ps}, 229.19 \mathrm{pJ}$, and $89.16 \mathrm{~W}$ in one polarization, respectively. In the other polarization, we can generate the dark soliton with 960 fs. Figures 2(c) and 2(d) show that dissipative and dark solitons remain stable when they circled 500 times in the cavity separately. According to Figure 2(d), we found that the dip exist not only in the pulse central but also in the edge, because in the simulation we used 


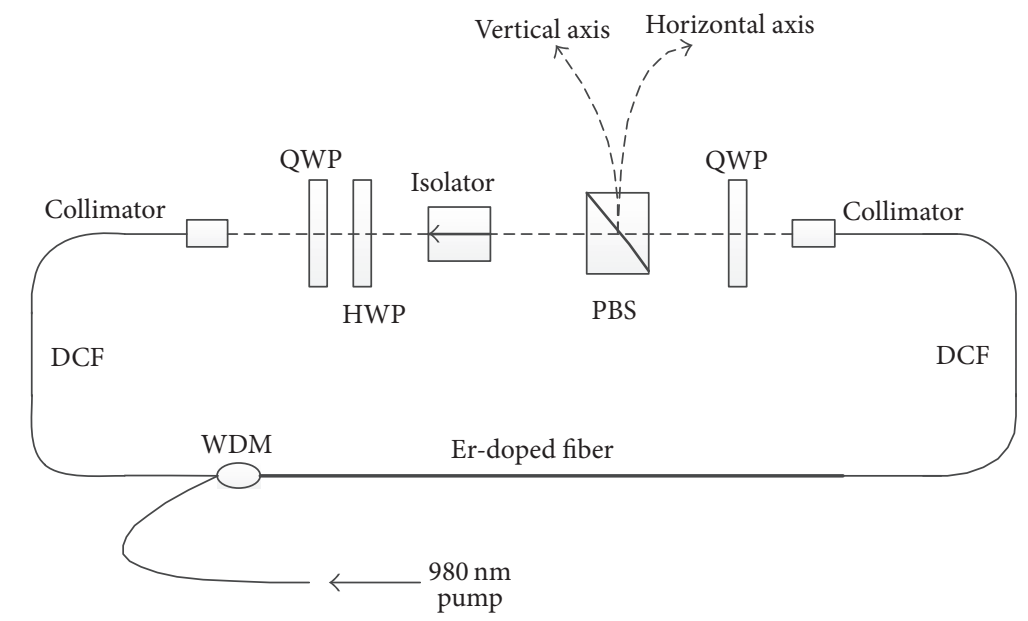

FIGURE 1: A schematic diagram of the all-normal dispersion fiber laser; PBS: polarization beam splitter; HWP: half waveplate; QWP: quarter waveplate; WDM: wavelength division multiplexer.

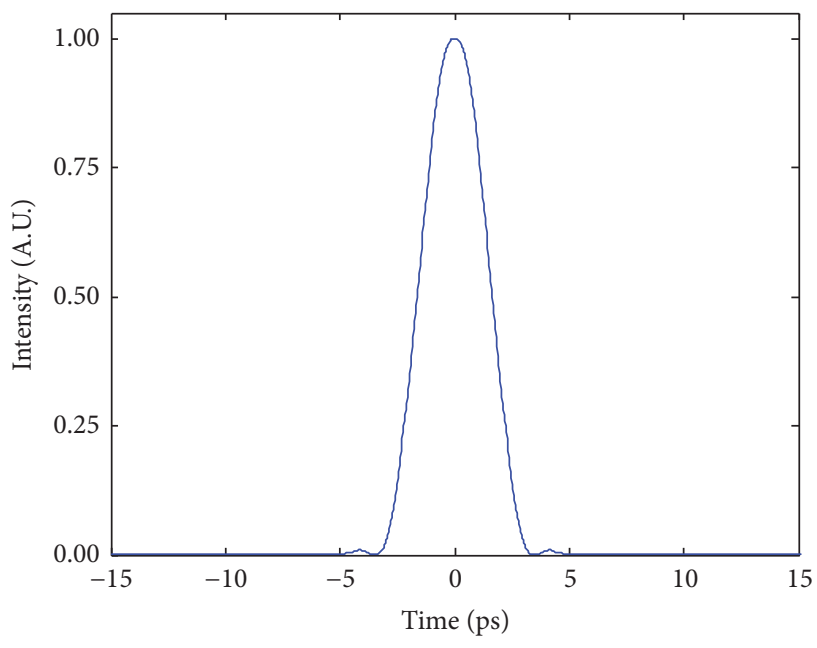

(a)

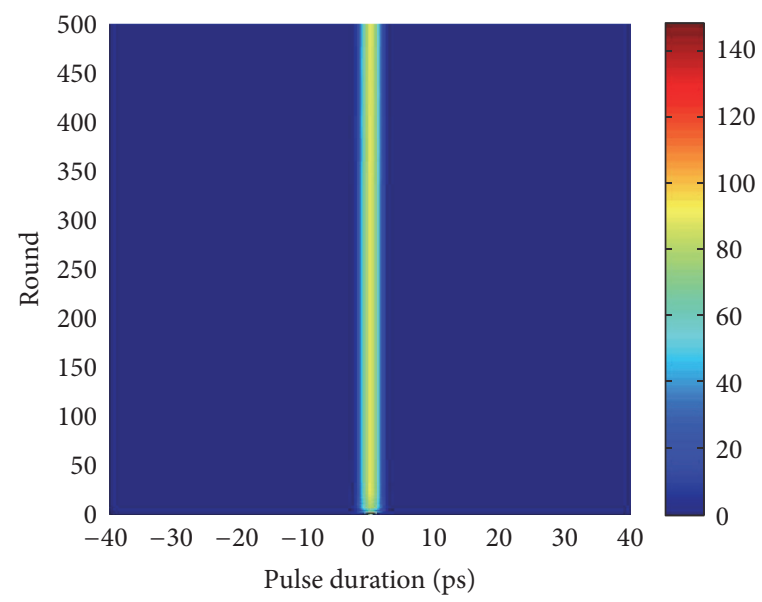

(c)

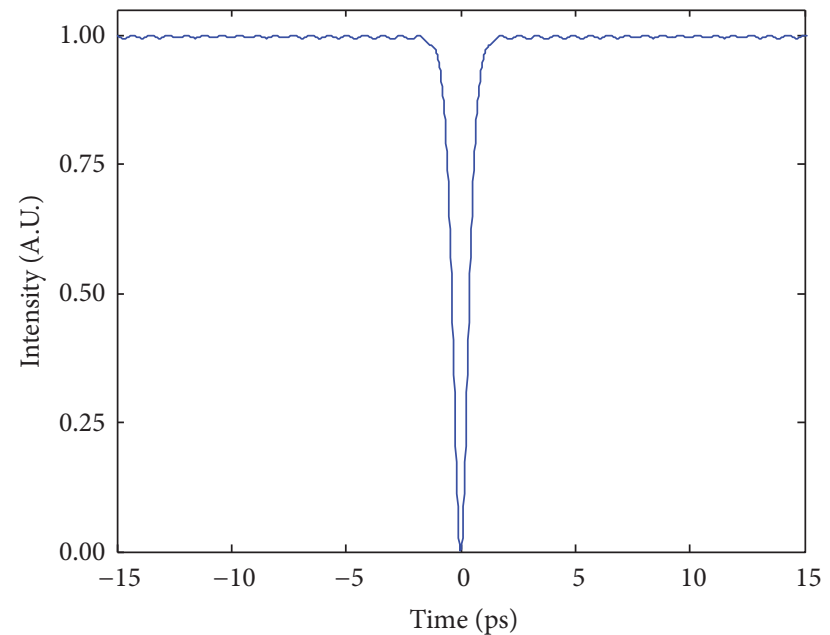

(b)

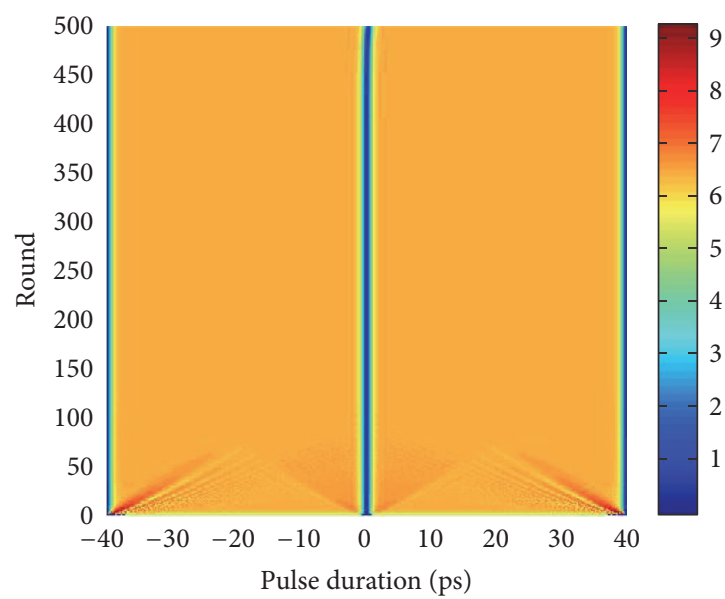

(d)

Figure 2: (a) Temporal profile of the dissipative soliton at $E_{\text {sat }}=460 \mathrm{pJ}$. (b) Temporal profile of the dark soliton at $E_{\text {sat }}=460 \mathrm{pJ}$. (c) Dynamic evolution of dissipative soliton. (d) Dynamic evolution of dark soliton. 


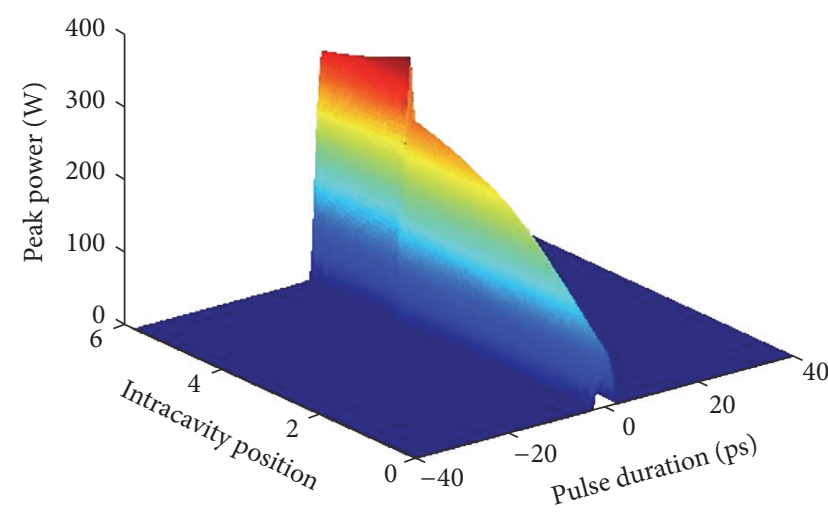

(a)

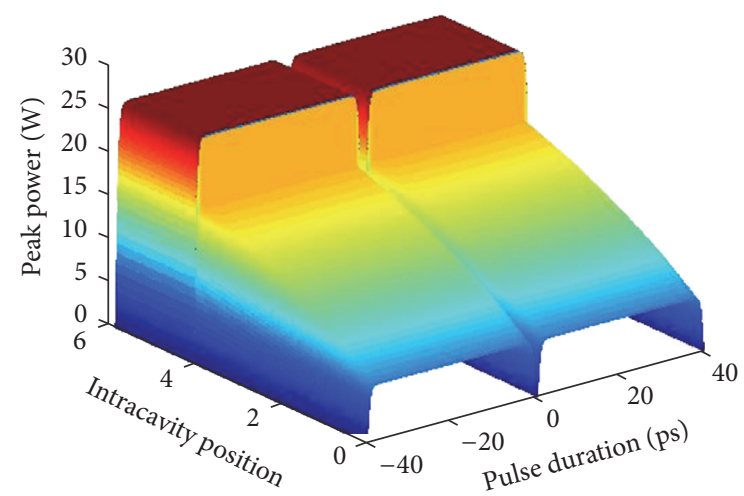

(b)

FIGURE 3: (a) Intracavity dissipative soliton evolution (at round =498) in temporal domain. (b) Intracavity dark soliton evolution (at round = 498) in temporal domain.

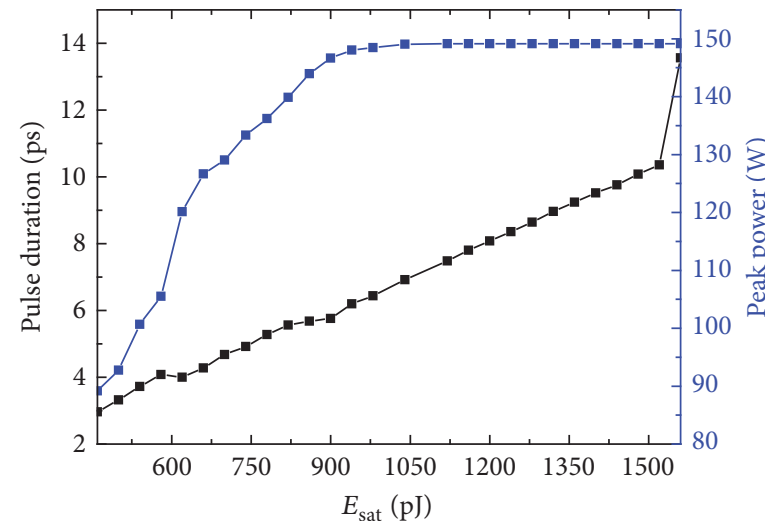

(a)

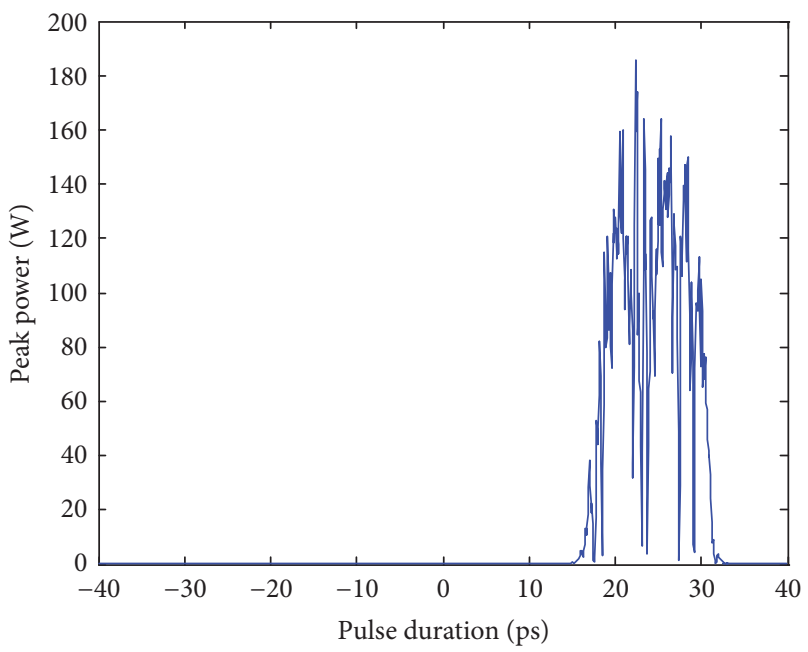

(c)

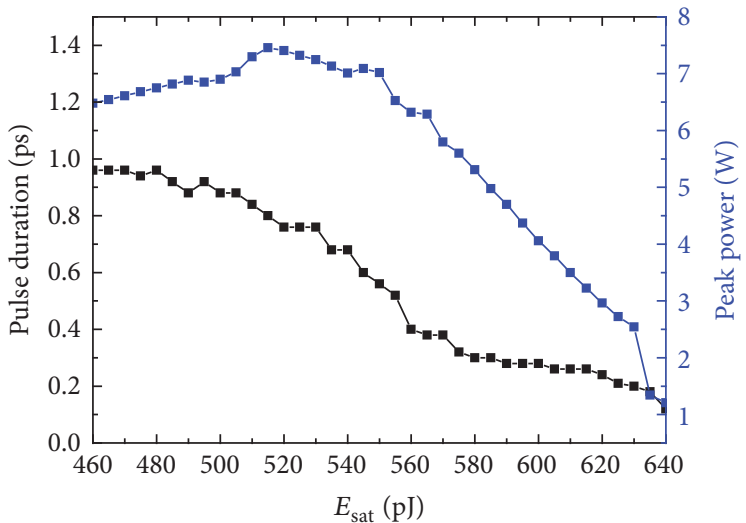

(b)

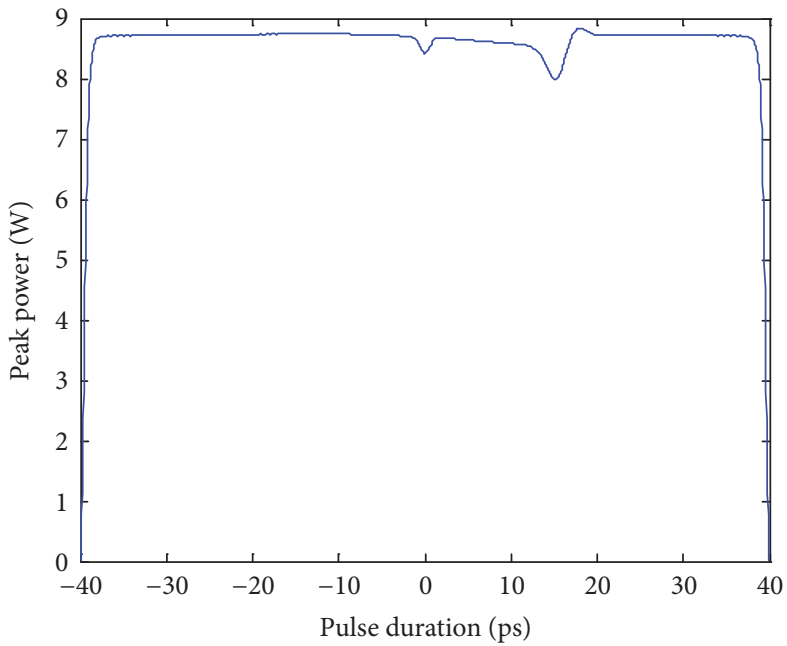

(d)

FIGURE 4: (a) With the increase of $E_{\text {sat }}$, dissipative soliton pulse duration and peak power change. (b) With the increase of $E_{\text {sat }}$, dark soliton pulse duration and peak power change. (c) Temporal profile of the dissipative soliton at $E_{\text {sat }}=1560 \mathrm{pJ}$. (d) Temporal profile of the dark soliton at $E_{\text {sat }}=640 \mathrm{pJ}$. 


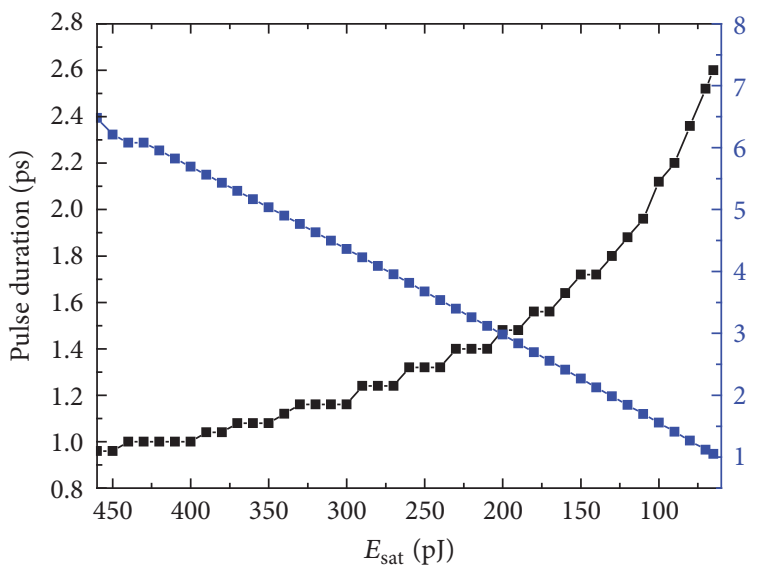

(a)

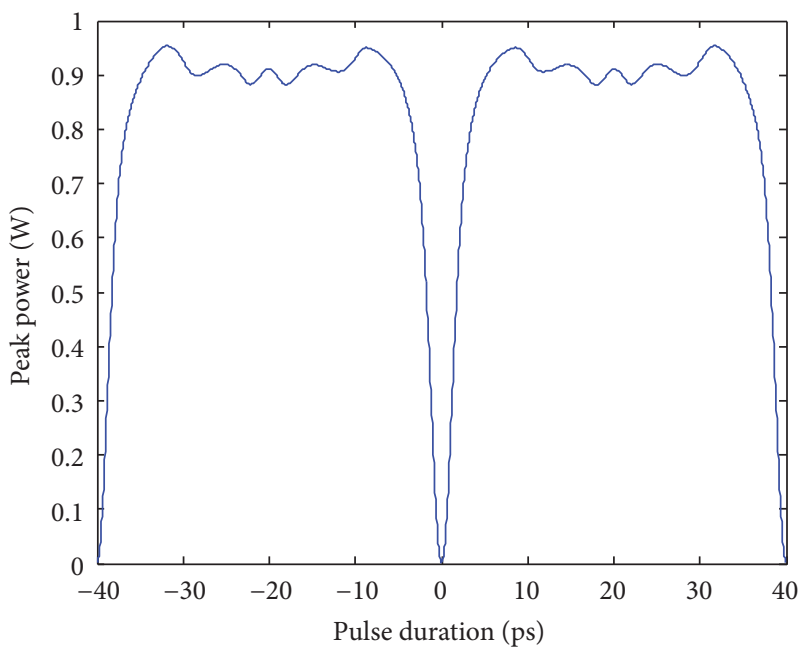

(c)

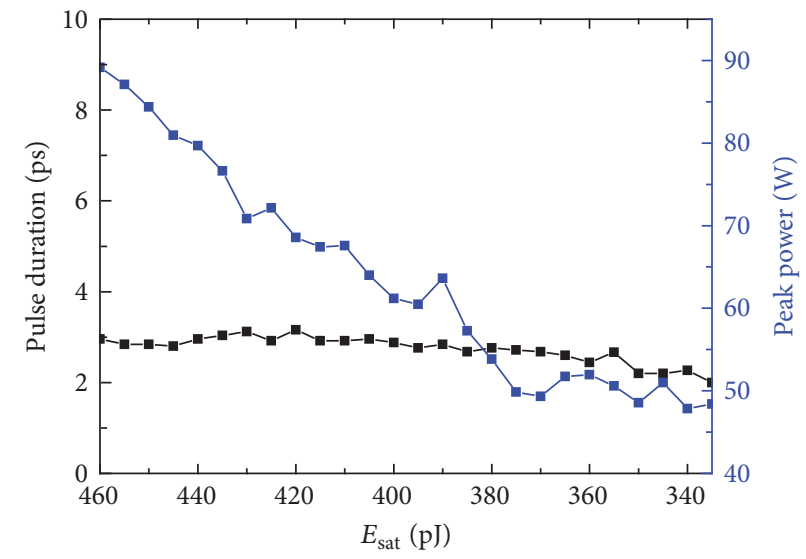

(b)

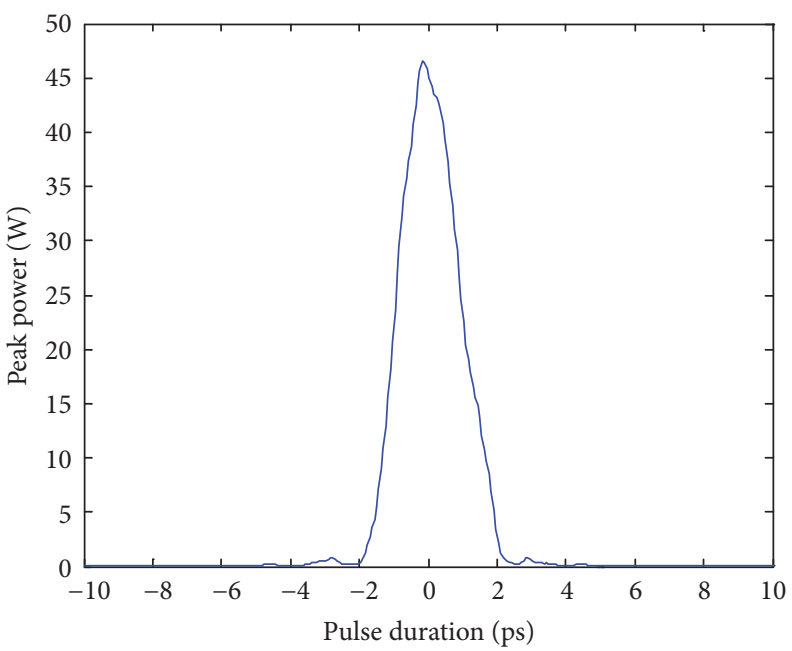

(d)

FiguRE 5: (a) With the decrease of $E_{\text {sat }}$, the dark soliton pulse duration and peak power change. (b) With the decrease of $E_{\text {sat }}$, the dissipative soliton pulse duration and peak power change. (c) Temporal profile of the dark soliton at $E_{\text {sat }}=58 \mathrm{pJ}$. (d) Temporal profile of the dissipative soliton at $E_{\text {sat }}=335 \mathrm{pJ}$.

the finite background pulse instead of infinite background pulse to generate dark soliton. Numerical simulation shows that if the background pulse width is 10 times the soliton width, the transmission characteristics of finite and infinite backgrounds of dark solitons are basically the same, so we can ignore the edge dip. From Figures 3(a) and 3(b) we can observe the impact of the various parts of the components for peak power and pulse duration. After propagating the EDF, both the dissipative soliton and the dark soliton peak power show an increase trend. However, as for the pulse duration, the dissipative soliton shows an increase while the dark soliton remains nearly stable. When soliton transmitted to PAPM, both dissipative and dark soliton pulse durations are rapidly compressed while their peak power remains the same. In the DCF, the dissipative soliton peak power related to the previous EDF has been increased rapidly and then decreased slowly, but it has no effect on the pulse duration. As for the dark soliton, the peak power in DCF increases rapidly and then remains unchanged, and the same basic has no effect on dark soliton pulse width. These results are obtained when $E_{\text {sat }}=460$ pJ. Next, we analyze the various $E_{\text {sat }}$ effects for our system.

Figure 4 shows the change of dissipative and dark solitons with the increase of $E_{\text {sat }}$. Figure 4(a) reflects the notion that dissipative soliton pulse duration and peak power increase with $E_{\text {sat }}$. When $E_{\text {sat }}$ increases to about $980 \mathrm{pJ}$, peak power is nearly unchanged, and when $E_{\text {sat }}$ reaches 1560 pJ, dissipative soliton appears split in Figure 4(c). If we continue to increase $E_{\text {sat }}$, the dissipative soliton will generate an unstable pulse which is different from the phenomenon adopted in Liu [2]. However, Figure 4(b) shows that dark soliton varies with different dissipative soliton; firstly the peak power increases with $E_{\text {sat }}$, but the dark soliton pulse duration gradually decreases. When $E_{\text {sat }}$ increases to $550 \mathrm{pJ}$, the peak power reduces gradually and eventually in $E_{\text {sat }}=640 \mathrm{pJ}$, the dark soliton disappears in Figure 4(d).

Figures 5(a) and 5(b) show that with the decrease of $E_{\text {sat }}$, both the dissipative soliton and the dark soliton peak power 
show a decrease trend. However, as for the pulse duration, the dark soliton shows an increase while the dissipative soliton remains nearly stable. When $E_{\text {sat }}$ is reduced to $335 \mathrm{pJ}$, the dissipative soliton is unstable. When $E_{\text {sat }}$ is below $318 \mathrm{pJ}$, the dissipative soliton cannot be generated. Then we conclude that the generation of dissipative soliton requires a pump power exceeding a threshold. When $E_{\text {sat }}$ is reduced to $58 \mathrm{pJ}$, the dark soliton is unstable, but it is still capable of forming a dark soliton. It can be found that obtaining a dark soliton does not require high $E_{\text {sat }}$, which means the dark soliton does not require high pump power.

\section{Conclusion}

We propose a novel way for controlling the generation of the dissipative bright soliton and dark soliton operation of lasers. Numerical simulation shows that, in an appropriate pump power range, the dissipative bright soliton and dark soliton can be generated simultaneously in the mode-locked and non-mode-locked regions, respectively. When $E_{\text {sat }}$ is up to $640 \mathrm{pJ}$, only dissipative soliton can be achieved, and the dissipative soliton is unstable when $E_{\text {sat }}$ is close to $1560 \mathrm{pJ}$, whereas it does not generate multipulse phenomenon [2]. Then if we decrease $E_{\text {sat }}$ to 335 pJ, only dark soliton can be obtained. Hence, we conclude that the dissipative soliton can tolerate higher pump power but its generation process requires a high threshold, whereas dark soliton can be obtained at a low pump power threshold.

\section{Competing Interests}

The authors declare that there are no competing interests regarding the publication of this paper.

\section{References}

[1] C. Zhao, H. Zhang, X. Qi et al., "Ultra-short pulse generation by a topological insulator based saturable absorber," Applied Physics Letters, vol. 101, no. 21, Article ID 211106, 2012.

[2] X. Liu, "Hysteresis phenomena and multipulse formation of a dissipative system in a passively mode-locked fiber laser," Physical Review A, vol. 81, no. 2, Article ID 023811, 2010.

[3] X. Wu, D. Y. Tang, H. Zhang, and L. M. Zhao, "Dissipative soliton resonance in an all-normal-dispersion erbium-doped fiber laser," Optics Express, vol. 17, no. 7, pp. 5580-5584, 2009.

[4] Y. Chen, C. Zhao, S. Chen et al., "Large energy, wavelength widely tunable, topological insulator Q-switched erbium-doped fiber laser," IEEE Journal on Selected Topics in Quantum Electronics, vol. 20, no. 5, pp. 315-322, 2014.

[5] H. Zhang, D. Y. Tang, L. M. Zhao, and X. Wu, "Observation of polarization domain wall solitons in weakly birefringent cavity fiber lasers," Physical Review B, vol. 80, no. 5, Article ID 052302, 2009.

[6] D. Li, D. Tang, L. Zhao, and D. Shen, "Mechanism of dissipativesoliton-resonance generation in passively mode-locked allnormal-dispersion fiber lasers," Journal of Lightwave Technology, vol. 33, no. 18, pp. 3781-3787, 2015.

[7] D. D. Han, X. M. Liu, Y. D. Cui, G. X. Wang, C. Zeng, and L. Yun, "Simultaneous picosecond and femtosecond solitons delivered from a nanotube-mode-locked all-fiber laser," Optics Letters, vol. 39, no. 6, pp. 1565-1568, 2014.
[8] X. Li, Y. Wang, W. Zhao et al., "Numerical investigation of soliton molecules with variable separation in passively modelocked fiber lasers," Optics Communications, vol. 285, no. 6, pp. 1356-1361, 2012.

[9] X. Li, X. Liu, X. Hu et al., "Experimental observations of separation-changeable soliton pairs in a fiber laser modelocked by nonlinear polarization rotation technique," Journal of Optoelectronics and Advanced Materials, vol. 13, no. 3, pp. 190195, 2011.

[10] W. H. Renninger, A. Chong, and F. W. Wise, "Self-similar pulse evolution in an all-normal-dispersion laser," Physical Review A, vol. 82, no. 2, Article ID 021805, 2010.

[11] X. Liu, "Mechanism of high-energy pulse generation without wave breaking in mode-locked fiber lasers," Physical Review A, vol. 82, no. 5, Article ID 053808, 2010.

[12] B. Guo, Y. Yao, P. G. Yan et al., "Dual-wavelength soliton modelocked fiber laser with a WS2-based fiber taper," IEEE Photonics Technology Letters, vol. 28, no. 3, pp. 323-326, 2016.

[13] X. Li, Y. Wang, W. Zhang, and W. Zhao, "Experimental observation of soliton molecule evolution in $\mathrm{Yb}$-doped passively modelocked fiber lasers," Laser Physics Letters, vol. 11, no. 7, Article ID 075103, 2014.

[14] Z.-C. Luo, W.-J. Cao, Z.-B. Lin, Z.-R. Cai, A.-P. Luo, and W.-C. $\mathrm{Xu}$, "Pulse dynamics of dissipative soliton resonance with large duration-tuning range in a fiber ring laser," Optics Letters, vol. 37, no. 22, pp. 4777-4779, 2012.

[15] D. Mao, X. M. Liu, L. R. Wang, X. H. Hu, and H. Lu, "Partially polarized wave-breaking-free dissipative soliton with superbroad spectrum in a mode-locked fiber laser," Laser Physics Letters, vol. 8, no. 2, pp. 134-138, 2011.

[16] X. Liu, "Dissipative soliton evolution in ultra-large normalcavity-dispersion fiber lasers," Optics Express, vol. 17, no. 12, pp. 9549-9557, 2009.

[17] F. Ö. Ilday, J. R. Buckley, W. G. Clark, and F. W. Wise, "Selfsimilar evolution of parabolic pulses in a laser," Physical Review Letters, vol. 92, no. 21, Article ID 213902, 2004.

[18] W. H. Renninger, A. Chong, and F. W. Wise, "Dissipative solitons in normal-dispersion fiber lasers: exact pulse solutions of the complex ginzburg-landau equation," in Proceedings of the Bragg Gratings, Photosensitivity, and Poling in Glass Waveguides, Quebec City, Canada, September 2007.

[19] H. Zhang, D. Y. Tang, L. M. Zhao, Q. L. Bao, and K. P. Loh, "Large energy mode locking of an erbium-doped fiber laser with atomic layer graphene," Optics Express, vol. 17, no. 20, pp. 17630 17635, 2009.

[20] X. Li, Y. Wang, W. Zhao et al., "All-fiber dissipative solitons evolution in a compact passively $\mathrm{Yb}$-doped mode-locked fiber laser," Journal of Lightwave Technology, vol. 30, no. 15, Article ID 6204196, pp. 2502-2507, 2012.

[21] B. Gao, J. Huo, G. Wu, and X. Tian, "Soliton molecules in a fiber laser mode-locked by a graphene-based saturable absorber," Laser Physics, vol. 25, no. 7, Article ID 075103, 2015.

[22] Z. Wang, S.-E. Zhu, Y. Chen et al., "Multilayer graphene for Q-switched mode-locking operation in an erbium-doped fiber laser," Optics Communications, vol. 300, pp. 17-21, 2013.

[23] X. Li, X. Liu, X. Hu et al., "Long-cavity passively mode-locked fiber ring laser with high-energy rectangular-shape pulses in anomalous dispersion regime," Optics Letters, vol. 35, no. 19, pp. 3249-3251, 2010.

[24] H. Zhang, D. Y. Tang, L. M. Zhao, X. Wu, and H. Y. Tam, "Dissipative vector solitons in a dispersion-managed cavity fiber 
laser with net positive cavity dispersion," Optics Express, vol. 17, no. 2, pp. 455-460, 2009.

[25] X. He, L. Hou, M. Li et al., "Bound States of dissipative solitons in the single-mode Yb-doped fiber laser," IEEE Photonics Journal, vol. 8, no. 2, pp. 1-7, 2016.

[26] Y. Q. Ge, J. L. Luo, L. Li et al., "Initial conditions for dark soliton generation in normal-dispersion fiber lasers," Applied Optics, vol. 54, no. 1, pp. 71-75, 2015.

[27] L. Li, F. Y. Song, H. Zhang et al., "Dark soliton operation fiber lasers," in Proceedings of the 2013 Conference on Lasers and Electro-Optics Pacific Rim (CLEO-PR '13), WPB_21, Optical Society of America, Kyoto, Japan, 2013.

[28] D. Y. Tang, L. Li, Y. F. Song, L. M. Zhao, H. Zhang, and D. Y. Shen, "Evidence of dark solitons in all-normal-dispersion-fiber lasers," Physical Review A, vol. 88, no. 1, Article ID 013849, 2013.

[29] T. P. Horikis and M. J. Ablowitz, "Constructive and destructive perturbations of dark solitons in mode-locked lasers," Journal of Optics, vol. 17, no. 4, Article ID 042001, 2015.

[30] H. Zhang, D. Y. Tang, L. M. Zhao, and X. Wu, "Dark pulse emission of a fiber laser," Physical Review A-Atomic, Molecular, and Optical Physics, vol. 80, no. 4, Article ID 045803, 2009.

[31] H. Zhang, D. Y. Tang, L. M. Zhao, and R. J. Knize, "Vector dark domain wall solitons in a fiber ring laser," Optics Express, vol. 18, no. 5, pp. 4428-4433, 2010.

[32] Y. Meng, S. Zhang, H. Li, J. Du, Y. Hao, and X. Li, "Brightdark soliton pairs in a self-mode locking fiber laser," Optical Engineering, vol. 51, no. 6, Article ID 064302, 2012.

[33] D. Tang, J. Guo, Y. Song, H. Zhang, L. Zhao, and D. Shen, "Dark soliton fiber lasers," Optics Express, vol. 22, no. 16, pp. 1983119837, 2014.

[34] K.-I. Maruno, A. Ankiewicz, and N. Akhmediev, "Dissipative solitons of the discrete complex cubic-quintic GinzburgLandau equation," Physics Letters A, vol. 347, no. 4-6, pp. 231240, 2005.

[35] A. Cabasse, B. Ortaç, G. Martel, A. Hideur, and J. Limpert, "Dissipative solitons in a passively mode-locked Er-doped fiber with strong normal dispersion," Optics Express, vol. 16, no. 23, pp. 19322-19329, 2008. 

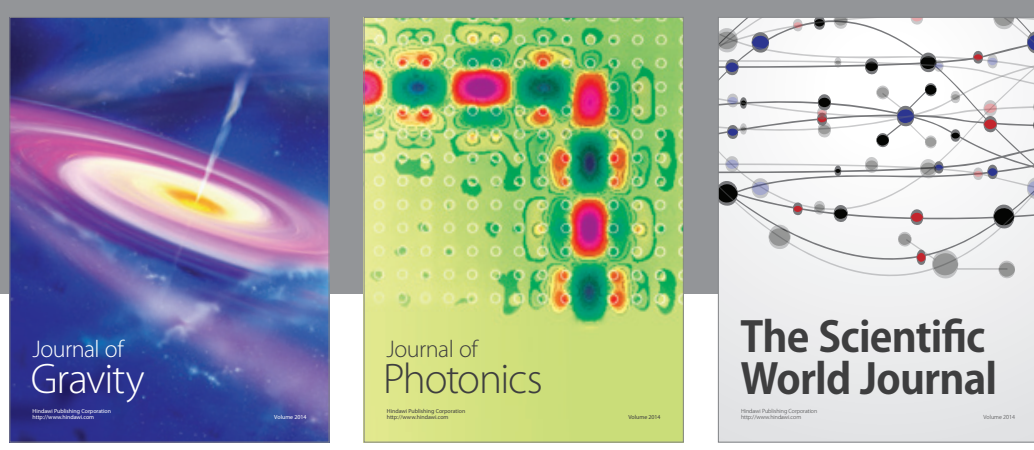

The Scientific World Journal
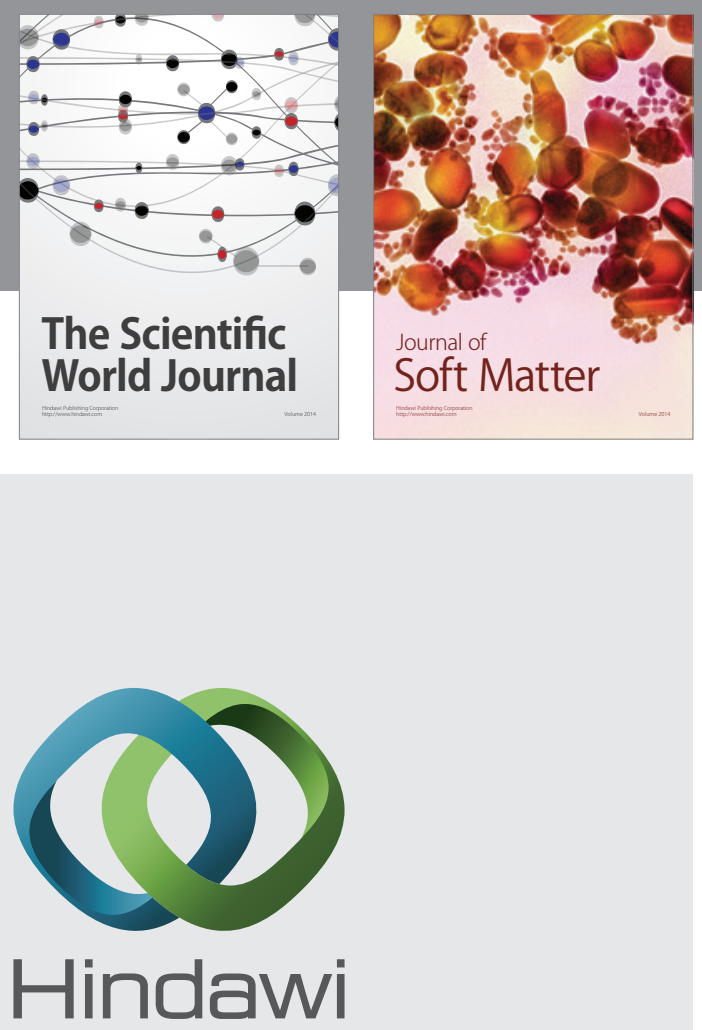

Submit your manuscripts at

http://www.hindawi.com

nternational Journal of

Statistical Mechanics
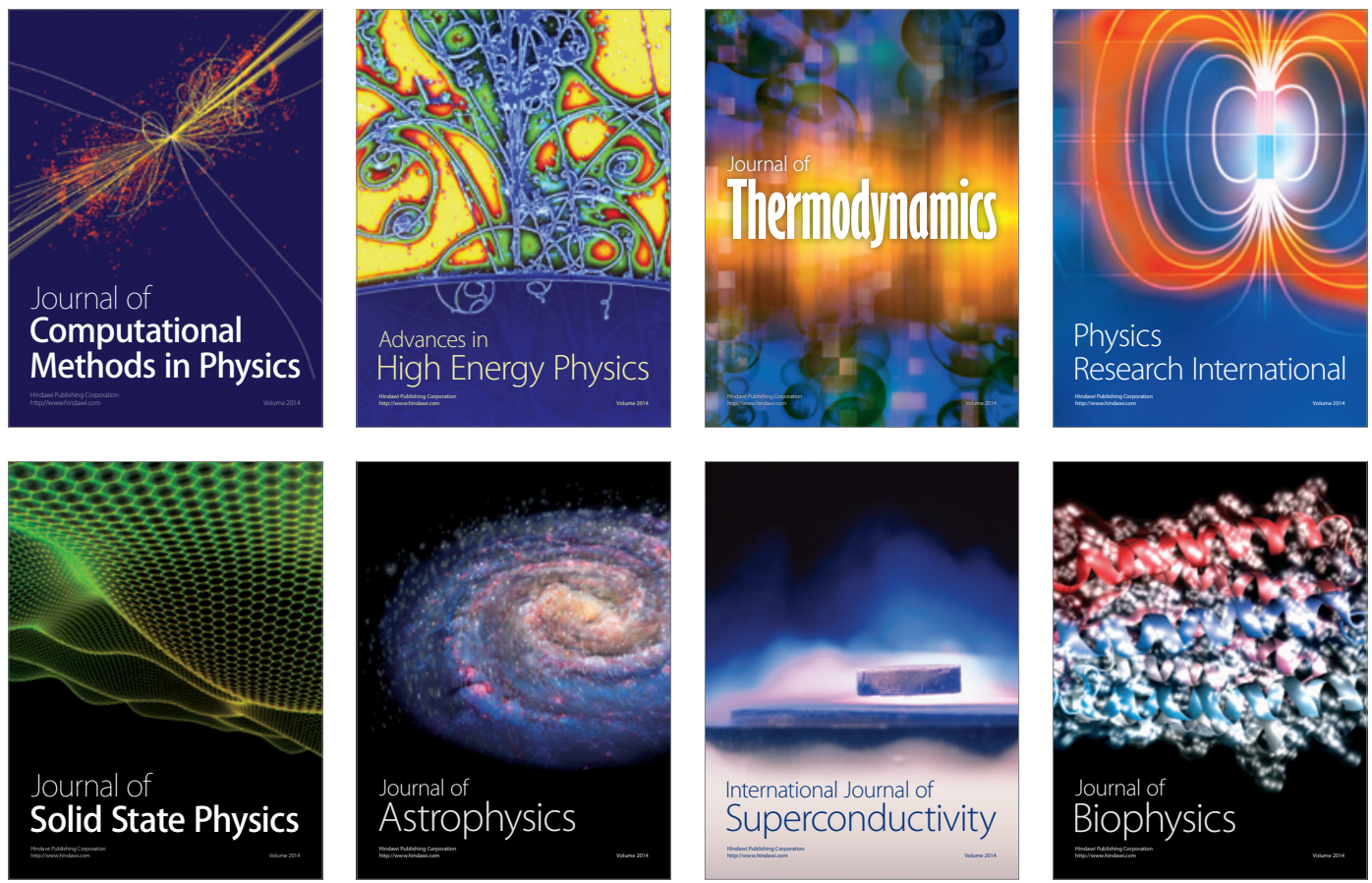
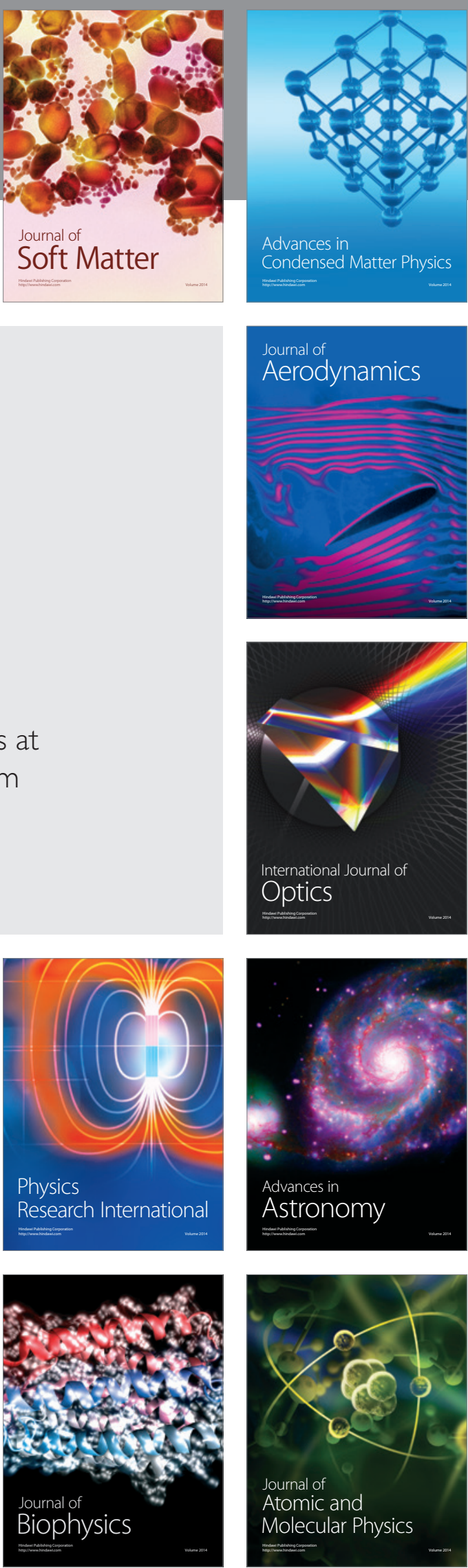